\title{
Parental control: ecology drives plasticity in parental response to offspring signals
}

Authors: Caro, S.M. ${ }^{1,2,3 *}$, Velasco, A.C. ${ }^{4}$, van Mastrigt, T. ${ }^{3,5}$, van Oers, K. ${ }^{5}$, Griffin, A.S. ${ }^{2}$, West, S.A. ${ }^{2}$, \& Hinde, C.A. ${ }^{6}$

\section{Affiliations:}

${ }^{1}$ Department of Integrative Biology, University of Texas at Austin, 2415 Speedway \#C0930, Austin, TX 78712, USA.

2 Department of Zoology, University of Oxford, Oxford OX1 3SZ, UK.

3 Behavioural Ecology Group, Department of Animal Sciences, Wageningen University and Research, PO Box 338, 6700 AH, Wageningen, The Netherlands.

${ }^{4}$ Department of Evolutionary Ecology, National Museum of Natural Sciences, Spanish National Research Council (MNCN-CSIC), 28006 Calle de José Gutiérrez Abascal 2, Madrid, Spain.

${ }^{5}$ Department of Animal Ecology, Netherlands Institute of Ecology (NIOO-KNAW), PO Box 50, $6700 \mathrm{AB}$, Wageningen, The Netherlands.

${ }^{6}$ Department of Biomedical and Forensic Science, Faculty of Science and Technology, Anglia Ruskin University, East Road, Cambridge, Cambridgeshire, CB1 1PT, UK.

*Corresponding author (email: Shana_Caro@post.harvard.edu)

\section{Abstract}

Different bird species have completely different parent-offspring interactions. When food is plentiful, the chicks that are begging the loudest are fed the most. When food is scarce, bird species

6 instead feed the largest offspring. While this variation could be due to parents responding to

7 signalling differently based on food availability, it could equally be due to offspring adjusting their

8 behaviour, or to variation in information availability. We tested between these competing

9 explanations experimentally, by manipulating food availability in a population of wild great tits, more plentiful, parents were: (1) more likely to preferentially feed the chicks that were begging

12 the most; and (2) less likely to preferentially feed larger chicks. In addition, we consistently found

13 these same patterns, in a meta-analysis across 57 bird species. Overall, our results suggest that 
15 how they respond to both offspring signals and cues of offspring quality in response to food

16 availability. Consequently, depending upon environmental conditions, predictably different

17 signalling systems are favoured.

18

Keywords: parent-offspring communication, signalling, plasticity, begging, meta-analysis, Parus major 


\section{Introduction}

20 In species where parents care for multiple offspring at the same time, families are constantly

21 negotiating how much parents will invest in each offspring. Yet the outcome of these negotiations

22 is completely different in different bird species (Caro et al. 2016). At one extreme, in some species,

23 the chicks in worse condition beg the most, and gain the most food from their parents. At the other

24 extreme, in other species, begging appears to be ignored, and the largest chicks obtain the most

25 food. Evolutionary theory and a comparative, across-species study have suggested that this pattern

26 reflects parents' adjusting their feeding strategy in response to environmental conditions and food

27 availability (Davis et al. 1999; Kilner 2002; Mock et al. 2011; Grodzinski and Johnstone 2012;

28 Caro et al. 2016; Koykka and Wild 2018). When food is plentiful, parents will be able to rear all

29 their offspring, and so should preferentially feed the offspring in greatest need, which can be

30 signalled by begging (Godfray 1995; Davis et al. 1999). In contrast, when food is scarce and only

31 a fraction of offspring can be raised, parents should preferentially feed the best quality offspring

32 based on size cues (Caro et al. 2016). This hypothesis posits that differences across species are

33 determined by parents adjusting their responses to signals in response to environmental conditions.

35 However, this hypothesis was based on observational data, and is open to alternative explanations.

36 First, changes in communication patterns could be controlled by either receivers (parents) or

37 signallers (offspring) (Kilner and Hinde 2008). If offspring control resource allocation via direct

38 competition, then the most competitive offspring should receive the most food. Consequently, in

39 situations where competitive ability shows greater variation, which could be when food is scarce,

40 we would expect signals of need to have less influence on food distribution (Royle et al. 2002;

41 Parker et al. 2002). Second, parents could have access to different information under different 
42 environmental conditions, which would constrain their ability to respond to signals (Kilner and

43 Hinde 2008). For example, if all offspring beg at maximum intensity in worse environments,

44 parents cannot use begging to distinguish between offspring. Third, it is only known that across-

45 species differences are correlated with environmental conditions-it is not known whether

46 individuals are plastic in how they communicate based on local conditions. We might only expect

47 plasticity to evolve in species that experience variable ecological conditions within or between

48 breeding bouts (Forsman 2015). If parents could flexibly vary their decision rules according to the

49 environment, that would substantially increase the level of control that parents have within the

50 family (Kilner and Hinde 2008).

52 We used an experimental approach to directly test whether parents respond differently to begging

53 depending upon food availability in great tits, Parus major. This species lives in temperate regions

54 and is exposed to variable breeding conditions across years, and could therefore be expected to

55 evolve plasticity in response to offspring signals and cues. To distinguish between parental and

56 offspring responses to food availability, we both provided supplemental food to some parents and

57 cross-fostered offspring before observing behaviour. This allowed us to observe parents that had

58 and had not been supplemented, interacting with foster broods that were similar in begging

59 intensity, chick size cues, chick competitive asymmetry and supplementation history, as foster

60 broods comprised half supplemented, half unsupplemented chicks. Next, to investigate whether

61 plasticity is a general pattern across species, we conducted a phylogenetic meta-analysis,

62 examining the pattern of behaviour within 57 bird species. We assessed within-species plasticity

63 by quantifying each species' change in responsiveness to begging or chick size as environmental

64 quality improves. 
Materials and Methods

Experimental study

\section{Study area and species}

68 Great tits (Parus major) are a common passerine bird distributed across Eurasia. They are

69 primarily insectivorous while feeding young, with highly variable food availability both

70 geographically and temporally (van Balen 1973). This variation in ecological conditions within

71 and between breeding bouts makes great tits a prime candidate for studying the evolution of

72 flexibility in parental provisioning strategies. We studied a wild population of great tits living in a

73 mixed pine-deciduous forest (Boslust) covering approximately 75 ha in The Netherlands $\left(5^{\circ} 85^{\prime} \mathrm{E}\right.$,

$\left.7452^{\circ} 01^{\prime} \mathrm{N}\right)$. From March through June 2017, we monitored 130 nest boxes, and were able to include

7534 broods in our study. We checked nest boxes every other day to determine the onset of egg

76 laying and clutch size. We began visiting nests daily the day before hatching was expected to

77 determine hatch date (day 0), brood size and mortality rates. Mean clutch size was 9.29 $\pm 0.23 \mathrm{SE}$

78 eggs, and mean brood size at hatching was $8.82 \pm 0.26 \mathrm{SE}$ in our study population. All of the study

79 broods hatched within 9 days of each other. Across all broods, $10.9 \%$ of chicks (33 of 302 chicks)

80 died in the first week after hatching.

81

\section{Experimental procedures}

83 In order to simulate variation in ecological conditions, we experimentally manipulated food

84 availability in an alternating pattern: half of the broods received supplemental food (mealworms

85 and wax worms), while the other half experienced natural conditions (see supplemental methods

86 for details). 
88 We wanted all parents to be exposed to equivalent information from their broods during filming,

89 so that we could rule out the possibility that offspring are driving any differences in parental

90 provisioning preferences. We therefore standardized brood size and offspring supplementation

91 history across all broods immediately before filming. We cross-fostered chicks on the filming day

92 (8 days after hatching) to create experimental filming broods of 7 (27 broods) or 6 chicks (4

93 broods). Approximately half of the chicks in each filming brood came from a supplemented nest,

94 and the other half of the chicks came from an unsupplemented nest. Fostered chicks were the same

95 age as the parents' biological brood. Parents were not filmed with their own chicks. We also

96 wanted to ensure that there would be sufficient and equivalent variation in offspring size so that

97 parents could use this information during food allocation. To create an even distribution of weight

98 and prior weight ranks in filming broods, we ranked chicks by weight in their biological nests. We

99 assigned the heaviest chick to filming brood A, and the second heaviest to filming brood B, the

100 third heaviest to brood A, etc. We alternated this pattern at each nest. We wanted to ensure that

101 there would be enough variation in begging intensity and to ensure initial begging intensity would

102 vary across weight ranks. We hand-fed half of the chicks in each filming brood to satiation, in an

103 alternating pattern by weight rank (see supplemental methods for details). This ensured that not all

104 chicks begged maximally during filming and that not all small chicks begged at highest intensity

105 the whole time.

106

107 Thus, parents in both treatments were filmed at the same time of day, feeding broods of an 108 equivalent size and begging intensity, comprising unrelated supplemented and unsupplemented 109 chicks, half of which were satiated when the filming began. 


\section{Details for great tit experimental supplementation}

112 To ensure only experimental broods received extra food, and to avoid changes to nest defense 113 associated with positioning the food near the nest box, we installed a small feeding tray inside each 114 nest box (Verhulst 1994; Grieco 2003; Eeva et al. 2009). This was done during incubation at all 115 nests. No broods were deserted after the introduction of the tray. Each day for the first week after 116 hatching, we provided a c. 20g mixture of live meal worms (Tenebrio molitor) and rehydrated wax 117 Worm larvae (Galleria mellonella) cut into $0.25 \mathrm{~cm}$ pieces to supplemented nests. This represents 118 approximately $20 \%$ of the daily nutritional needs of the brood (van Balen 1973; Eeva et al. 2009).

119 We checked whether great tits were using the food by placing cameras into 2 nests during the 120 supplementation period. We observed parents taking food from the trays and directly feeding their

121 offspring (Supplementary Movie 2), and parents also ate the food themselves. Either outcome 122 serves to increase environmental conditions for the parents. Control nests were also visited each 123 day so that all nests received comparable experimental disturbance, and an empty tray was placed 124 in the nest box.

126 We alternated experimental treatments by assigning the first brood of the day that had hatchlings

127 to the supplemented treatment, and then the next brood the unsupplemented (control) treatment.

128 We reversed this order each day. We did not pre-randomize because we wanted to equalise hatch 129 date within each treatment. Supplemented and unsupplemented nests varied slightly in clutch size 130 (supplemented $9.81+/-0.33 \mathrm{se}$, unsupplemented $8.82+/-0.32, \mathrm{p}=0.038^{*}$ ), but not in brood size 131 (supplemented 9.18 +/- 0.36se, unsupplemented $8.59+/-0.36, \mathrm{p}=0.26$ ) or hatch date 132 (supplemented 25.29+/- 0.58se, unsupplemented 25.18+/- 0.57, $\mathrm{p}=0.89$ ). The difference in clutch 
133 size was driven by one unsupplemented nest with only 6 eggs; removing this nest or including

134 clutch size as a control variable did not change the results of our parental response model.

\section{Details of cross-fostering and hand-feeding}

137 All cross-fostering was done in the morning as soon as possible prior to filming, and all filming 138 occurred between 7:00 and 15:00 (83\% of feeding visits occurred between 9:00 and 13:00).

140 Hand-feeding protocol: We ranked chicks by weight in their filming nests. We assigned chicks to

141 be handfed or not handfed in an alternating pattern by weight rank, which was reversed at each

142 nest. For example, in filming brood A, the heaviest chick was handfed and the second heaviest was

143 not, while in filming brood B the heaviest chick was not handfed. Immediately prior to filming,

144 we hand-fed chicks in an artificial nest containing a cloth wrapped hand-warmer. We fed the 145 selected chicks with Nutribird A 19 high energy bird food using a $5 \mathrm{~mL}$ syringe. We continued 146 feeding until begging had ceased and could no longer be induced by whistling and tapping the 147 sides of the bill with a syringe, indicating the chicks were probably satiated, as in (Kilner and 148 Davies 1998).

\section{Video data}

151 We filmed parents feeding their foster broods 8 days after hatching (see Supplementary Movie 1

152 for an example of video data). We installed an infrared camera inside the lid of a nest box the day 153 prior to filming in order to habituate parents. We paint-coded all chicks with a dot of red, non154 toxic acrylic paint on the head (Kate Lessells, pers. comm.) just prior to filming, so that we could 155 individually identify chicks in the videos. We excluded the first 30 minutes of filming to ensure 
that parental and chick behaviour had enough time to return to normal after cross-fostering and to

157 give us enough time to leave the area. We did not provide supplemental food to the parents on the 158 filming day.

All videos were coded by the same observer, blind to the experimental treatment and to chick weight ranks. The order in which the observer coded the videos was random with respect to

162 whether nests were supplemented and unsupplemented. Adult identity was determined by the

163 difference in crown feather glossiness of males and females, and confirmed by nest cleaning 164 behaviour, which only females perform (Christe et al. 1996). For each feeding visit, the observer 165 recorded the sex of parent, the identity of the fed chick, and the begging intensity of all chicks. 166 The observer recorded 20 feeding visits per parent or 4 hours of filming, whichever came first.

\section{Begging intensity}

169 Begging intensity was coded on a standard scale, following Hinde 2009, adapted from Kilner 170 (1995): 0 = non-gaping, 1 = gaping with a bent neck, 2 = gaping with neck stretched out, $3=$ 171 gaping with raised body (Kilner 1995; Hinde et al. 2009). We quantified relative begging intensity

172 by dividing the begging posture of each chick by the mean posture of all begging chicks on that

173 feeding visit. Values greater than 1 indicate that parents preferentially fed chicks with a higher

174 posture score than their nest mates. This relative measure accounts for differences in overall 175 begging intensity on different feeding visits, which could confound measures of food distribution 176 based on absolute begging intensity (Hinde et al. 2009).

\section{Chick size}


179 We ranked chicks by weight in their filming brood, with chick 1 being the heaviest and chick 7

180 being the lightest. Using weight rank as opposed to absolute weight makes nests more directly

181 comparable - parents may always prefer feeding the largest chick, whether the largest chick

182 weighs $12 \mathrm{~g}$ or $10 \mathrm{~g}$. A priori, we assumed that weight rank need not have a linear effect. Parents

183 may treat large and medium sized chicks differently than they treat small chicks, since the smallest

184 chicks may be most vulnerable to starvation (Magrath 1990; Forbes et al. 1997; Amundsen and

185 Slagsvold 1998; Theofanellis et al. 2008; Podlas et al. 2013). Furthermore, the difference in

186 absolute weight between ranks was lower in the middle of hierarchy $(0.68 \mathrm{~g})$ than at either end

187 (1.05g). We therefore included the quadratic term for weight rank in analyses.

\section{Statistical analysis}

190 We checked whether our supplementation treatment was biologically relevant by investigating its

191 effect on the likelihood of brood reduction (whether at least one chick died) in the first week after

192 hatching before any cross-fostering took place. The effect of supplementation on likelihood of

193 brood reduction likelihood was assessed using a binomial linear model in lme4 in R, while

194 controlling for clutch size, brood size, and hatch date (Bates et al. 2015). The extent of brood

195 reduction was assessed using a quasi-poisson linear model to account for zero-inflated count data,

196 while controlling for clutch size, brood size, and hatch date (Bates et al. 2015). Chick mass one

197 week after hatching was assessed using a linear mixed model, while controlling for clutch size,

198 hatch date, and nest ID (Bates et al. 2015). We standardised and centered control variables (Cohen 199 et al. 2003). 
201 We analysed parental provisioning using a Bayesian logistic mixed model (MCMCglmm) in R

202 (Hadfield 2010; R Core Team 2013). We used uninformative priors, ran the model for 700,000

203 iterations with a burn-in of 150,000 and a thinning interval of 10 . We assessed the convergence of

204 the MCMCglmm model by visual inspection of convergence plots and geweke plots (Hadfield

$2052010 ;$ 2012). The response variable was whether a chick was fed or not. We included nest ID,

206 parent ID, chick ID, and feeding visit ID as random effects. We analysed a three-way interaction

207 between supplementation treatment, relative begging intensity, and weight rank as the fixed effect.

208 We were interested in this interaction because our main hypothesis was about the moderating effect

209 of supplementation, and because parents may respond differently to begging of different offspring

210 (van Heezik and Seddon 1996). We tested whether weight rank had a non-linear effect. We

211 included both the linear and quadratic 3-way interaction terms in the same model, along with all

212 possible 2-way interactions using both the linear and quadratic terms (Ganzach 1997). If the

213 quadratic interactions were not significant, we would have removed them. Including polynomial

214 terms in interactions can lead to false positives or negatives due to collinearity (Ganzach 1997).

215 Centering variables reduces this collinearity between polynomials, and so we scaled and centred

216 begging and weight rank (Cohen et al. 2003; Dalal and Zickar 2011).

218 Of the 34 broods filmed for our study, we excluded one brood because the parents abandoned 219 during filming, and two broods that had fewer than 20 feeding visits. We excluded data from four 220 parents with fewer than 15 observed feeding visits. We excluded six feeding visits where the 221 begging posture of more than two chicks was unknown. Our final sample size for the analysis of 222 parental provisioning was 14 supplemented nests, 15 unsupplemented nests (54 adults, 199 chicks, 
2231121 feeding visits). We analysed the full data set as well, and there were no qualitative differences

224 in the results or in their statistical significance.

225

226 Comparative study

227 Data collection for the meta-analysis

228 To determine whether birds show a consistent adjustment of feeding rules based on local

229 conditions across species, we collected data on within-species changes in the strength of the

230 relationship (correlation coefficient) between feeding and begging in 17 species, and feeding and

231 size cues in 52 species (719 effect sizes from 145 studies; Data S1; Fig. S1). We conducted a

232 literature search on Web of Science and Google Scholar using the keywords 'beg', 'parent-

233 offspring', 'bird', 'begging', ‘communication' and 'provision' (see Fig. S1 for PRISMA flowchart

234 detailing data collection and exclusion criteria). We included all papers with any measure relating

235 to the relationship between parental food allocation and offspring behavioural begging or size cues.

236 We excluded species that did not have data on these relationships in more than one environment

237 condition, since we were interested in the change in the strength of these relationships over

238 different ecological conditions. We excluded studies if it was impossible to determine whether

239 parents were responding to begging or to size cues. We excluded studies where offspring signals

240 were structural (such as mouth colour), rather than behavioural (such as begging postures), as these

241 may represent different signalling systems (Caro et al. 2016). There was not enough within-species

242 data on structural signals in different environments include them. We only included effect sizes

243 for the relationship of begging on within-brood food allocation, rather than on increases in overall

244 parental feeding effort, as these represent fundamentally different aspects of parental care. We

245 excluded data on species that lay only one egg per brood, as selective pressures on these species 
246 are likely to differ from species laying multiple eggs per brood. If relevant data were given in

247 papers without statistical tests, such as raw means and standard errors, we estimated effect sizes

248 (Borenstein et al. 2011). This resulted in a dataset of 719 effect sizes from 145 studies on 57 species

249 (Data S1).

251 Environmental quality

252 We categorized populations as experiencing normal, better than normal, or worse than normal

253 environments, based on experimental manipulations of long-term chick condition (parents were

254 fed reduced or supplemented diets, or chick demand was artificially increased or decreased),

255 ecological measures (such as prey density, date or rainfall), or average offspring mortality across

256 different years in long-term observational studies (Caro et al. 2016). These measures were not

257 always directly related to food availability, but they likely captured variation in some ecological

258 aspect relevant to offspring condition. If no information on environmental quality was available,

259 data were classified as normal conditions.

261 Measures of feeding, begging and size cues

262 Many aspects of behavioural begging were reported in the literature, such as begging amplitude,

263 duration, latency, likelihood, call structure, and begging postures. Different measures of food

264 allocation were also presented, such as weight gain over a short time period, actual food intake,

265 number of food items received, likelihood of being fed, growth rate, and mortality. We assumed

266 all measures of begging intensity and feeding preferences were functionally equivalent, and so

267 included all reported statistics in our analyses. Because test statistics were converted to a

268 standardized scale, differences between the various measures of begging intensity or feeding 
269

270

271 feeding preference was used or whether studies were experimental or observational, on the effect

272 size of begging or size cues on feeding preferences (Caro et al. 2016).

\section{Statistical analyses}

275 We transformed any test statistic measuring either an effect of begging or size cues on feeding into 276 a standardized effect size (Fisher's Z-transformed correlation coefficient) (Borenstein et al. 2011;

277 Grissom and Kim 2011; Koricheva et al. 2013). These correlation coefficients follow a normal

278 distribution, account for different scales in their original measurements, are well suited to the

279 ordered nature of the data, and are more straightforward to interpret than standardized differences

280 in means (Borenstein et al. 2011; Grissom and Kim 2011; Koricheva et al. 2013). Fisher's Z-

281 transformed correlation coefficients were analysed using the MCMCglmm package in R, which

282 implements Bayesian generalized linear mixed models with Markov chain Monte Carlo methods

283 (Hadfield 2010; R Core Team 2013). We ran bivariate response models so we could measure

284 phylogenetic covariance between responsiveness to begging and responsiveness to size cues. We

285 weighted models by sample size, and controlled for phylogeny and repeated measures on the same

286 study and species. Our measure of sample size was the number of broods used to generate the

287 original test statistic, because this is a standard measure across studies, and conservatively avoids

288 pseudoreplication if chick number or number of observations were used as the sample size. We

289 treated environmental quality as a three-level ordered categorical variable. To control for

290 phylogeny, we obtained phylogenies from Birdtree.org, ran models on 4 random phylogenetic trees

291 with Ericson and Hackett backbones, and then averaged model results (Jetz et al. 2014). 


\section{Results}

\section{Great tit experiment}

295 We found that parental provisioning rules were flexible in response to environmental conditions 296 parents responded differently to offspring begging and size, depending upon whether parents had

297 received supplemental food in the previous week (interaction between supplementation, quadratic 298 weight rank and relative begging intensity: 95\% CI -1.09 to -0.03 , pMCMC =0.041*; Fig. 1; Table 299 S2). In all nests and for all chicks, the likelihood of being fed increased with higher relative 300 begging intensity (95\% CI 1.91 to 2.87, pMCMC $<0.0001 * * *$; Table S2), but chick size mediated

301 how much begging increased the likelihood of being fed. In nests that had been supplemented (Fig.

302 1a), parents responded primarily to begging signals, responding equally to the begging of all chicks

303 regardless of size. However, in unsupplemented nests (Fig. 1b), parents responded more to size by 304 responding more to the begging of larger chicks than to the begging of the smallest chicks.

We confirmed that this difference in food allocation could not be explained by differences in offspring behaviour or size cues. Since we swapped chicks between nests directly before filming, there was no difference in relative begging posture or in chick weights between supplemented and unsupplemented foster broods (mean of begging: $\mathrm{t}_{27}=0.63, \mathrm{p}=0.53$; SD of begging: $\mathrm{t}_{27}=0.15, \mathrm{p}$

$310=0.88$; mean of weight: $\mathrm{t}_{27}=-0.90, \mathrm{p}=0.38$; SD of weight: $\left.\mathrm{t}_{27}=-0.64, \mathrm{p}=0.53\right)$. Since we handfed

311 a subset of chicks across the weight hierarchy, relative begging posture also did not vary by chick

312 weight rank $\left(\mathrm{t}_{167.8}=0.40, \mathrm{p}=0.69\right)$ or quadratic weight rank $\left(\mathrm{t}_{174.2}=-0.62, \mathrm{p}=0.54\right)$. We also

313 confirmed that our supplemental feeding was successful in improving environmental conditions:

$31459 \%$ of supplemented nests had no brood reduction in the first week after hatching, compared to 
315 only $18 \%$ of unsupplemented control nests $\left(\mathrm{z}=2.94, \mathrm{p}=0.0033^{* *} ; \mathrm{n}=34\right.$ nests; Table S1). The 316 total number of chicks that died per nest was also lower in supplemented nests $(\mathrm{z}=2.10, \mathrm{p}=$ $3170.045^{*}$; Table S1). Chick mass of the surviving chicks on day 7 was not affected by 318 supplementation $(\mathrm{z}=1.58, \mathrm{p}=0.12$; Table $\mathrm{S} 1)$.

\section{Comparative study}

321 Next, to explore whether plasticity is a general trend across birds, we conducted a phylogenetic 322 meta-analysis on 57 bird species. Species were included if they had data on parental responsiveness 323 to begging or chick size in multiple environmental conditions (poor, normal or good). We 324 quantified responsiveness as the correlation coefficient (effect size) between feeding and (a) 325 begging or (b) size. These two coefficients vary between $+/-1$, with higher values indicating that 326 either (a) begging or (b) size has a stronger effect on the likelihood that a chick is fed. We estimated 327 plasticity by calculating each species' change in responsiveness as environmental quality varies, 328 i.e. the within-species slope of correlation coefficients over environmental quality. A positive slope 329 would indicate that parents become more responsive in better environments, a slope of 0 would 330 indicate no plasticity based on the environment, and a negative slope would indicate that parents

331 become less responsive in better environments. If other species adjust their behaviour in the way 332 that we have observed with great tits, then we would observe a consistent within-species pattern, 333 with parents becoming more responsive to begging (positive slopes), and less responsive to size 334 (negative slopes), in better environments.

336 We found this predicted pattern, with parents became more responsive to begging, and less 337 responsive to size, in better environments. Specifically, we found a stronger correlation between 
338

339

340

341

342

343

344

345

346

347

348

349

350

351

352

353

354

355

356

357

358

359

360

begging and feeding in better environmental conditions $(95 \% \mathrm{CI}$ of the slope $=0.13$ to 0.67 , pMCMC $=0.0037$; Fig. 2a). Across 17 species with available data on responsiveness to begging in more than one environmental condition, 14 species showed the predicted increase in correlation strength (82\%), and three species showed a decrease (18\%).

Also, as predicted, we found the opposite pattern in parental response to size cues. Within species, there was a weaker correlation between chick size and feeding in better environmental conditions $(95 \% \mathrm{CI}$ of the slope $=-0.23$ to $-0.05, \mathrm{pMCMC}=0.0016 ;$ Fig. $2 \mathrm{~b})$. Across 52 species with available data on responsiveness to size cues in more than one environmental condition, 32 species showed the predicted decrease in correlation strength (62\%), and 20 had an increase (38\%). There was no species-level correlation between how responsive parents are to begging and how responsive they are to size cues $(95 \% \mathrm{CI}-0.54$ to 0.52$)$. These results suggest that the pattern we observed in great tits, where parents facultatively adjusted their responsiveness in reaction to local conditions, occurs consistently across a range of different bird species.

\section{Discussion}

Our experimental and comparative results show that parents conditionally adjust how they respond to signalling, depending upon environmental conditions (food availability). Parents did not simply feed the chicks that were the largest or that begged the most. Instead, they have evolved to adjust their sensitivity to multiple sources of information depending on local conditions, in a sophisticated manner. When food is more plentiful, great tit parents respond equitably to all their offspring's begging, but when food is more scarce, parents selectively respond more to the begging of larger chicks (Fig. 1a,b). Likewise in our meta-analysis, we found the same consistent pattern, 
across 57 species (Fig. 2a,b). These results show how variation in environmental quality can lead to different forms of communication, even within species.

364 The degree to which parents actively control food allocation as opposed to passively respond to 365 the greatest stimulus or cede to the winner of sibling competition has been contentious (Clutton-

366 Brock 1991; Kacelnik et al. 1995; Parker et al. 2002; Heeb et al. 2003; Ploger and Medeiros 2004).

367 Since our cross-fostering experiment ensured there was minimal variation in brood size,

368 competitive asymmetry and begging behaviour, changes in allocation patterns can definitively be 369 attributed to changes in great tit parental response strategies, rather than differences in offspring 370 behaviour or information constraints. It should be noted, however, that we examined provisioning 371 at one point midway through the nestling period. It is possible that older chicks may be able to 372 exert more control via scramble competition. Furthermore, given that species differed in the degree 373 of their plasticity (Fig. 2a,b), it is probable that species vary in the actual balance of power between 374 parents and offspring, so that in some species offspring behaviour drives changes in food 375 distribution patterns. It may be also that some species flexibly determine their investment strategies 376 in other ways and at other times; for example, 1) during incubation by varying the amount of 377 hatching asynchrony (e.g. blackbirds (Magrath 1992) and European rollers (Parejo et al. 2015)); 378 2) during different points in the breeding season by varying how parents respond to UV signals 379 (e.g. alpine swifts and European starlings (Bize et al. 2006)); or 3) during different times within a 380 single breeding attempt by varying aggression towards offspring (e.g. American coots (Shizuka 381 and Lyon 2012)). Recent work on genetic covariance and plasticity in canaries found that both 382 offspring and parental signalling strategies varied plastically across different hunger levels 383 (Fresneau and Müller 2019). This indicates that even if parents are plastic in their behavior and 
384 control provisioning, they may still be influenced by changes in their offspring's behavior. Future

385 research could continue disentangling what is driven by parental preference, by parents' reactions

386 to offspring signals, or by offspring directly.

387

388 What explains diversity in signalling systems is a fundamental question in signalling theory. Our

389 results suggest that receivers control the outcome of parent-offspring communication and assess

390 multiple sources of information from signallers. This is analogous to how females respond to

391 multiple signals of quality in sexual signalling (Bro-Jørgensen 2010), and may be similar to

392 aggressive signalling and other forms of communication as well. Our results highlight the need for

393 dynamic signalling models that allow for flexibility in responsiveness based on environmental

394 conditions, and that incorporate multiple signals and cues (Mangel and Clark 1988; Wild 2011). 
Figures and legends

A
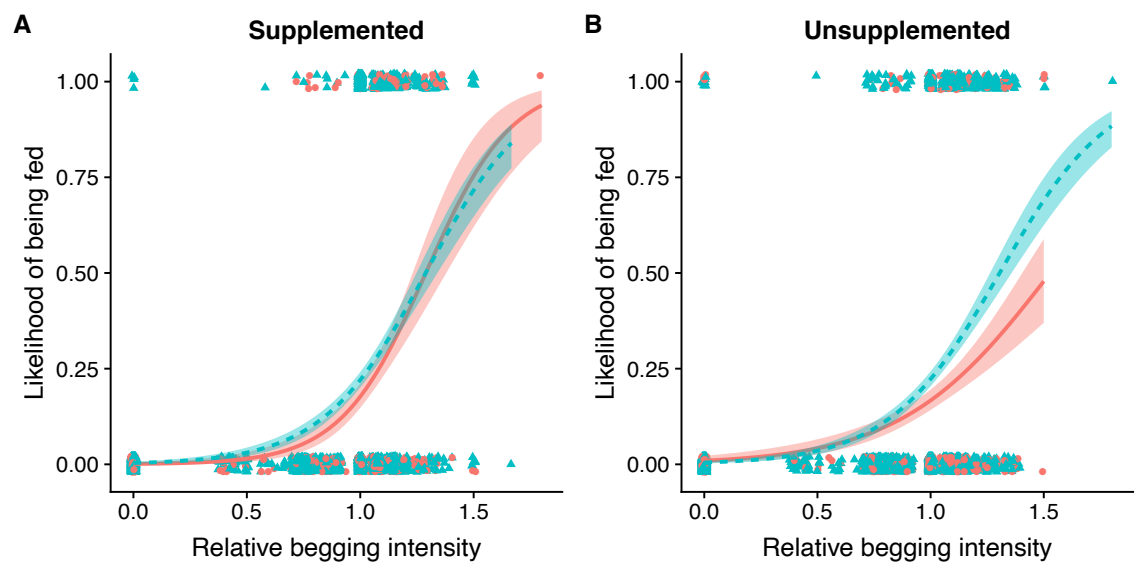

Fig. 1. A chick's likelihood of being fed depends on its relative begging intensity, size rank, and whether its parents were supplemented. In supplemented nests (a), higher relative begging intensity led to a greater likelihood of being fed for all chicks equally. In unsupplemented nests (b), smaller chicks showed less of an increase in their likelihood of being fed based on begging intensity than larger chicks did $(95 \% \mathrm{CI}$ of the interaction $=-1.09$ to $-0.03, \mathrm{MCMCglmm})$. Weight ranks 1-5 are shown in blue, while the smallest two chicks in the nest (ranks 6-7) are shown in red. We show weight categories for graphical clarity; statistical analyses report the non-linear effect of weight rank as a continuous variable. A relative begging intensity of 1 indicates a chick is begging the same as its nest mates on average, while $>1$ means it begged at a higher intensity. Each data point is one chick in a feeding visit, vertically jittered to show overlapping points ( $\mathrm{n}=14$ supplemented nests, 15 unsupplemented nests, 1121 feeding visits). 


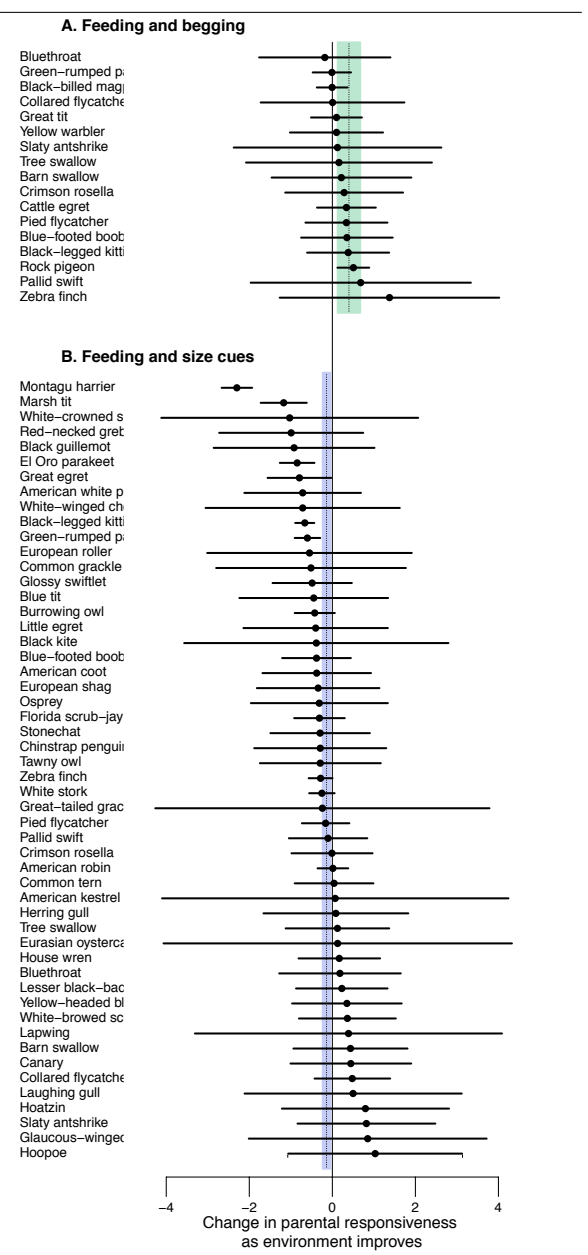

410 Fig. 2. Environmental quality and parental response to offspring begging and size. Circles

411 represent each species' slope for the correlation coefficient between feeding and (a) begging, and

412 (b) size cues, over environmental quality. Lines represent the 95\% CI of the slope within each

413 species. The shaded region shows the 95\% CI across all species, controlling for phylogeny and

414 weighted by sample size. Positive values indicate that parents respond more in better environments

415 (green). Negative values indicate parents respond less in better environments (purple). Species

416 respond more to begging $(95 \% \mathrm{CI}=0.13$ to $0.67, \mathrm{MCMCglmm}, \mathrm{n}=17$ species), and less to size

417 cues $(95 \% \mathrm{CI}=-0.23$ to -0.05 , MCMCglmm, $\mathrm{n}=52$ species $)$, in better environments. This pattern

418 is more consistent for changes in responsiveness to begging, but is also significant for changes in

419 responsiveness to size. 


\section{References:}

421 Amundsen, T., and T. Slagsvold. 1998. Hatching asynchrony in great tits: a bet-hedging 422 strategy? Ecology 79:295-304. Wiley-Blackwell.

423 Bates, D., M. Maechler, B. Bolker, and S. Walker. 2015. Fitting Linear Mixed-Effects Models 424 Using lme4. Journal of Statistical Software 67:1-48.

425 Bize, P., R. Piault, B. Moureau, and P. Heeb. 2006. A UV signal of offspring condition mediates 426 context-dependent parental favouritism. Proc. R. Soc. B 273:2063-2068.

427 Borenstein, M., L. V. Hedges, J. P. T. Higgins, and H. R. Rothstein. 2011. Introduction to Meta428 Analysis. John Wiley \& Sons, Sussex, UK.

429 Bro-Jørgensen, J. 2010. Dynamics of multiple signalling systems: animal communication in a 430 world in flux. Trends Ecol. Evol. 25:292-300.

431 Caro, S. M., A. S. Griffin, C. A. Hinde, and S. A. West. 2016. Unpredictable environments lead 432 to the evolution of parental neglect in birds. Nature Communications 7:1-10. Nature Publishing 433 Group.

434 Christe, P., H. Richner, and A. Oppliger. 1996. Of great tits and fleas: sleep baby sleep ... 435 Anim. Behav. 52:1087-1092.

436 Clutton-Brock, T. 1991. The evolution of parental care. Oxford University Press, Princeton, NJ.

437 Cohen, J., P. Cohen, S. G. West, and L. S. Aiken. 2003. Applied multiple correlation/regression 438 analysis for the behavioral sciences. UK: Taylor \& Francis.

439 Dalal, D. K., and M. J. Zickar. 2011. Some Common Myths About Centering Predictor Variables 440 in Moderated Multiple Regression and Polynomial Regression. Organizational Research 441 Methods 15:339-362.

442 Davis, J. N., P. M. Todd, and S. Bullock. 1999. Environment quality predicts parental 443 provisioning decisions. Proc. R. Soc. B 266:1791-1797.

444 Eeva, T., S. Sillanpää, and J. P. Salminen. 2009. The effects of diet quality and quantity on 445 plumage colour and growth of great tit Parus major nestlings: a food manipulation experiment 446 along a pollution gradient. Journal of Avian Biology 40:491-499. Wiley/Blackwell (10.1111).

447 Forbes, S., S. Thornton, B. Glassey, M. Forbes, and N. J. Buckley. 1997. Why parent birds play 448 favourites. Nature 390:351-352.

449 Forsman, A. 2015. Rethinking phenotypic plasticity and its consequences for individuals, 450 populations and species. Heredity 115:276-284. Nature Publishing Group. 
451 Fresneau, N., and W. Müller. 2019. Flexible communication within bird families-The 452 consequences of behavioral plasticity for parent-offspring coadaptation. Ecol Evol 9:693-702.

453 John Wiley \& Sons, Ltd.

454 Ganzach, Y. 1997. Misleading interaction and curvilinear terms. Psychological Methods 2:235455247.

456 Godfray, H. C. J. 1995. Signaling of Need Between Parents and Young: Parent-Offspring 457 Conflict and Sibling Rivalry. Am Nat 146:1-24.

458 Grieco, F. 2003. Greater food availability reduces tarsus asymmetry in nestling blue tits. The 459 Condor 105:599-603.

460 Grissom, R. J., and J. J. Kim. 2011. Effect Sizes for Research: Univariate and Multivariate 461 Applications; 2nd ed. Taylor \& Francis, Hoboken, NJ.

462 Grodzinski, U., and R. A. Johnstone. 2012. Parents and offspring in an evolutionary game: the 463 effect of supply on demand when costs of care vary. Proc. R. Soc. B 279:109-115. The Royal 464 Society.

465 Hadfield, J. 2012. MCMCglmm Course Notes. Unpublished Manuscript.

466 Hadfield, J. D. 2010. MCMC Methods for Multi-Response Generalized Linear Mixed Models: 467 The MCMCglmm R Package. Journal of Statistical Software 33:1-22.

468 Heeb, P., T. Schwander, and S. Faoro. 2003. Nestling detectability affects parental feeding 469 preferences in a cavity-nesting bird. Anim. Behav. 66:637-642.

470 Hinde, C. A., K. L. Buchanan, and R. M. Kilner. 2009. Prenatal environmental effects match 471 offspring begging to parental provisioning. Proc. R. Soc. B 276:2787-2794. The Royal Society.

472 Jetz, W., G. H. Thomas, J. B. Joy, D. W. Redding, K. Hartmann, and A. O. Mooers. 2014. 473 Global distribution and conservation of evolutionary distinctness in birds. Curr. Biol. 24:919474930.

475 Kacelnik, A., P. A. Cotton, L. Stirling, and J. Wright. 1995. Food Allocation among Nestling 476 Starlings: Sibling Competition and the Scope of Parental Choice. Proceedings of the Royal 477 Society B: Biological Sciences 259:259-263. The Royal Society.

478 Kilner, R. 1995. When do Canary Parents Respond to Nestling Signals of Need? Proc. R. Soc. B $479260: 343-348$.

480 Kilner, R. M. 2002. Sex differences in canary (Serinus canaria) provisioning rules. Behav Ecol 481 Sociobiol 52:400-407.

482 Kilner, R. M., and C. A. Hinde. 2008. Information warfare and parent-offspring conflict.

483 Advances in the Study of Behavior 68:283-336. 
484 Kilner, R., and N. B. Davies. 1998. Nestling mouth colour: Ecological correlates of a begging 485 signal. Anim. Behav. 56:705-712.

486 Koricheva, J., J. Gurevitch, and K. Mengersen. 2013. Handbook of Meta-analysis in Ecology and 487 Evolution. Princeton University Press, Princeton, USA.

488 Koykka, C., and G. Wild. 2018. The influence of environmental variance on the evolution of 489 signaling behavior. Beh. Ecol. 29:814-820.

490 Magrath, R. D. 1990. Hatching asynchrony in altricial birds. Biol Rev Camb Philos Soc 65:587491622.

492 Magrath, R. D. 1992. Roles of Egg Mass and Incubation Pattern in Establishment of Hatching 493 Hierarchies in the Blackbird (Turdus merula). The Auk 109:474-487. American Ornithologists' 494 Union.

495 Mangel, M., and C. W. Clark. 1988. Dynamic modeling in behavioral ecology. Princeton 496 University Press, Princeton, NJ.

497 Mock, D. W., M. B. Dugas, and S. A. Strickler. 2011. Honest begging: expanding from Signal of 498 Need. Beh. Ecol. 22:909-917.

499 Parejo, D., J. M. Avilés, and M. Expósito. 2015. Hatching Asynchrony and Spring Climatic 500 Conditions in the European Roller. Evol Biol 42:443-451. Springer US.

501 Parker, G. A., N. J. Royle, and I. R. Hartley. 2002. Begging scrambles with unequal chicks: 502 interactions between need and competitive ability. Ecol Lett 5:206-215.

503 Ploger, B. J., and M. J. Medeiros. 2004. Unequal food distribution among great egret Ardea 504 albanestlings: parental choice or sibling aggression? Journal of Avian Biology 35:399-404.

505 Podlas, K. A., H. Richner, 2013. 2013. The adaptive function of hatching asynchrony: an 506 experimental study in great tits. Anim. Behav. 86:567-576.

507 R Core Team. 2013. R: A language and environment for statistical computing. R Foundation for 508 Statistical Computing. Vienna, Austria.

509 Royle, N. J., I. R. Hartley, and G. A. Parker. 2002. Begging for control: when are offspring 510 solicitation behaviours honest? Trends Ecol. Evol. 17:434-440.

511 Shizuka, D., and B. E. Lyon. 2012. Family dynamics through time: brood reduction followed by 512 parental compensation with aggression and favouritism. Ecol Lett 16:315-322.

513 Theofanellis, T., E. Galinou, and T. Akriotis. 2008. The role of hatching asynchrony in brood 514 size reduction of the great tit Parus major in a Mediterranean pine forest. Journal of Natural 515 History 42:375-380. 
516 van Balen, J. H. 1973. A Comparative Study of the Breeding Ecology of the Great Tit Parus 517 major in Different Habitats. The Condor 61:1-93.

518 van Heezik, Y. M., and P. J. Seddon. 1996. Scramble feeding in jackass penguins: within-brood 519 food distribution and the maintenance of sibling asymmetries. Anim. Behav. 51:1383-1390.

520 Verhulst, S. 1994. Supplementary Food in the Nestling Phase Affects Reproductive Success in 521 Pied Flycatchers (Ficedula hypoleuca). The Auk 111:714-716.

522 Wild, G. 2011. Direct fitness for dynamic kin selection. J. Evol. Biol. 24:1598-1610. Blackwell 523 Publishing Ltd. 